\title{
Associations of serum sex hormone binding globulin with bone mineral densities and higher 10-year probability of fractures in postmenopausal women with type 2 diabetes mellitus
}

\author{
Yixuan Jing ${ }^{1 \#}$, Xiaofeng Wang ${ }^{1,2 \#}$, Jingjia Yu ${ }^{1}$, Xiaojing Wang ${ }^{1}$, Yanman Zhou ${ }^{1}$, Bei Tao ${ }^{1}$, Lihao Sun ${ }^{1}$, \\ Jianmin Liu ${ }^{1}$, Hongyan Zhao ${ }^{1}$ \\ ${ }^{1}$ Department of Endocrine and Metabolic Diseases, Rui Jin Hospital, Shanghai Jiao Tong University School of Medicine, Shanghai Institute of \\ Endocrine and Metabolic Diseases, Shanghai Clinical Center for Endocrine and Metabolic Diseases, Shanghai 200025, China; ${ }^{2}$ Currently at Tong \\ Ren Hospital, Shanghai Jiao Tong University School of Medicine, Shanghai 200336, China \\ Contributions: (I) Conception and design: Y Jing, X Wang, J Liu, H Zhao; (II) Administrative support: J Liu; (III) Provision of study materials \\ or patients: Y Jing, X Wang, J Yu, X Wang; (IV) Collection and assembly of data: Y Jing, X Wang, Y Zhou, B Tao, L Sun; (V) Data analysis and \\ interpretation: Y Jing, X Wang; (VI) Manuscript writing: All authors; (VII) Final approval of manuscript: All authors. \\ \#These authors contributed equally to this work. \\ Correspondence to: Jianmin Liu, MD, PhD; Hongyan Zhao, MD, PhD. Department of Endocrine and Metabolic Diseases, Rui Jin Hospital, Shanghai \\ Jiao Tong University School of Medicine, Shanghai Institute of Endocrine and Metabolic Diseases, Shanghai Clinical Center for Endocrine and \\ Metabolic Diseases, No. 197 Rui-jin Er Road, Shanghai 200025, China. Email: ljm10586@rjh.com.cn; hyanzhao@163.com.
}

Background: Postmenopause and type 2 diabetes mellitus (T2DM) are associated with higher fracture risk. Sex hormones are important in maintaining woman skeleton health. The relationships of sex hormone(s) with bone mineral density (BMD) and fracture risk are still unclear in diabetic-postmenopausal women. This study aimed to investigate the relationships of sex hormones with BMDs and fracture risk in postmenopausal women with T2DM.

Methods: Two hundred and fourteen postmenopausal women with T2DM were included. BMDs at lumbar spine (L2-4), femoral neck (FN) and total hip (TH) were measured by dual-energy X-ray absorptiometry (DXA). The 10-year probability of fractures was accessed by modified fracture risk algorithm (FRAX) tool. Serum concentrations of sex hormones were measured.

Results: Sex hormone binding globulin (SHBG) was a determinant of BMDs at L2-4 $(\beta=-0.199, \mathrm{P}<0.05)$, TH $(\beta=-0.233, \mathrm{P}<0.05)$, major osteoporotic fracture (MOF) $(\beta=0.253, \mathrm{P}<0.001)$ and hip fracture $(\mathrm{HF})$ $(\beta=0.262, \mathrm{P}<0.001)$. Per SD increase in SHBG caused a $2 \%$ increase in the risk of osteoporosis/osteopenia. SHBG in quartile-4 was associated with 4.21 higher risk of osteoporosis/osteopenia compared with SHBG in quartile-1.

Conclusions: In postmenopausal women with T2DM, higher serum SHBG tended to be associated with lower BMDs, and increased the risk of osteoporosis/osteopenia and the fracture risk.

Keywords: Bone mineral density (BMD); sex hormone binding globulin (SHBG); type 2 diabetes mellitus (T2DM); postmenopause

Submitted May 15, 2019. Accepted for publication Aug 05, 2019.

doi: $10.21037 / \mathrm{atm} .2019 .08 .46$

View this article at: http://dx.doi.org/10.21037/atm.2019.08.46 


\section{Introduction}

Osteoporosis, a disease characterized by deteriorated microarchitecture and increased fragility of the bone, has a high prevalence in the postmenopausal women, leading to reduced quality of life, greater economic burden, elevated disability and even higher mortality $(1,2)$. Postmenopausal bone loss has long been ascribed to the deficiency of endogenous estradiol (E2) production with age (3). In recent years, lower testosterone $(\mathrm{T})$ and higher sex hormone binding globulin (SHBG) concentrations have also been recognized as factors related to increased bone loss and fracture risk in postmenopausal women $(4,5)$.

It has widely been reported that type 2 diabetes mellitus (T2DM) is associated with higher skeletal fragility and consequently increased fracture risk, despite the conflicting evidence that patients with T2DM show decreased, normal or even elevated bone mineral densities (BMDs) assessed by dual X-ray absorptiometry (DXA) compared with individuals without diabetes (6-8). The discrepancy might be attributed to the different examining sites of BMDs, different sites may result in various outcomes, especially in T2DM patients (9). A recent study reports the reduced bone mass and increased vertebral fractures in Chinese women with T2DM, indicating that BMD may still be used to reflect the bone health in diabetic females (10). To date, most studies exploring the relationships among sex hormones and skeleton have been conducted in nondiabetic subjects. It is necessary to better understand the relationships among sex hormones and BMDs at various sites in postmenopausal women with T2DM who have greater risks for osteoporosis and fractures.

World Health Organization (WHO) Fracture Risk Algorithm (FRAX) is an online instrument that has been widely used for the assessment of fracture risk by many professional organizations (3). FRAX employs clinical risk factors such as age, body mass index (BMI), previous fractures, and other factors alone or combined with femoral neck (FN) BMD determined by DXA to estimate the 10-year probability of major osteoporotic fracture (MOF) and hip fracture (HF) (11). There is evidence showing that FRAX may underestimate the future fracture risk in T2DM patients, because diabetic risk factors are not included in this tool $(12,13)$. Replacement of rheumatoid arthritis (RA) with DM in FRAX is recommended to effectively improve the performance of FRAX in T2DM $(14,15)$.

The present study aimed to explore the associations of sex hormones including SHBG, follicle-stimulating hormone (FSH), luteinizing hormone (LH), E2, total testosterone ( $\mathrm{T}$ ) and free testosterone (FT) with BMDs, osteoporosis/osteopenia and 10-year probability of MOF and HF determined with modified FRAX after the replacement of RA with T2DM in postmenopausal women with T2DM.

\section{Methods}

\section{Study design and participants}

The clinical information was retrospectively collected from postmenopausal women with T2DM who were hospitalized in the Department of Endocrine and Metabolic Diseases, Rui-jin Hospital, Shanghai Jiao-tong University School of Medicine between January 2010 and December 2012. Amenorrhea for more than one year in women aged $>50$ years was defined as postmenopause (16). Subjects with hormone treatment or endocrine diseases that may influence bone metabolism including Cushing syndrome, hyperthyroidism, hypogonadism were excluded; subjects with RA, chronic kidney disease (CKD) stages 4 and 5 (eGFR $<30 \mathrm{~mL} / \mathrm{min}$ ) and malignancy were excluded; subjects receiving pharmacotherapy that may apparently affect bone metabolism including alfacalcidol, calcitonin and bisphosphonates were excluded. Finally, a total of 214 postmenopausal women (aged 50-84 years) with T2DM were included in this study. The protocol for the research project was approved by the Ethics Committee of Rui-jin Hospital affiliated to Shanghai Jiao-tong University School of Medicine (2019 No. 45). All procedures performed in studies were in accordance with the ethical standards of the Institutional Research Board and with the Helsinki Declaration.

\section{Collection of demographic characteristics and anthropometric information}

The medical information was collected based on the medical records. Height was measured to $0.1 \mathrm{~cm}$, and weight recorded to $0.1 \mathrm{~kg}$. BMI was calculated as weight in kilograms divided by height in meters squared.

\section{Laboratory examinations}

Blood samples were collected in the morning after fasting for 10 hours. Glycated hemoglobin A1c (HbA1c) was measured using the hemoglobin testing system (Variant II, 
Bio-Rad, Hercules, CA). The serum parathyroid hormone (PTH) and sex hormones including SHBG, FSH, LH, $\mathrm{E} 2, \mathrm{~T}$ and FT were tested by chemical luminescence assay (Architech i2000sr, Abbott, Chicago, IL). The total cholesterol, triglyceride, high-density lipoprotein cholesterol (HDL-C), low-density lipoprotein cholesterol (LDL-C), creatinine (Cr) and uric acid (UA) were measured by an automatic biochemical analyzer (Modular E170, Roche, Basel, Switzerland). Serum 25(OH)D was detected by electrochemiluminescence immune-assay (CobasE601, Roche).

\section{BMD measurement and fracture risk assessment}

The BMDs at the lumbar spine (L2-4), FN and total hip (TH) were measured by DXA (Lunar Expert-1313, Lunar Corp, Madison, WI). The 10-year probability of fractures was determined with the modified FRAX tool (https://www. sheffeld.ac.uk/FRAX/tool.aspx? country=2), with following parameters: age, sex, weight, height, fracture history, parental history of hip fractures, glucocorticoid usage, RA (T2DM in the present study), smoking status and alcohol intake. A China-specific FRAX algorithm with FN BMD was selected to evaluate the 10 -year probability of MOF and $\mathrm{HF}$.

\section{Statistical analysis}

Continuous variables were compared with the Shapiro-Wilk test. Data with normal distribution are presented as mean \pm standard deviation (SD), data with abnormal distribution as median (interquartile) and categorical variables as number (percentage). Independent-sample t-test was employed to compare the variables with normal distribution and MannWhitney $U$ test to compare the variables with abnormal distribution. Spearman correlation was used to assess the correlations among sex hormones, BMDs and 10-year probability of MOF and HF. Multivariate linear regression analysis was performed to determine the contributions of sex hormones to the BMDs at three sites, MOF and HF. Considering the original FRAX design, risk factors such as age and BMI were not enrolled in linear analysis. In logistic regression models, important sex hormones were fitted as continuous variables to examine the odds ratios (ORs) and $95 \%$ confidence interval (CI) of osteoporosis/osteopenia (defined as a BMD T-score at either L2-4, FN or TH less than -1). Receiver operating characteristic curves (ROC) of osteoporosis/osteopenia were generated to evaluate the maximal Youden index and the associated cut-off values of SHBG. In multivariate logistic regression model, the ORs (95\% CI) of osteoporosis/osteopenia across cut-off values of SHBG and potential risk factors were calculated. The prevalence rate and ORs (95\% CI) of osteoporosis/ osteopenia according to the quartiles of SHBG were compared with Chi-square test and logistic regression, respectively. Scatter diagrams with trend lines of SHBG concentrations with BMDs and MOF, HF were produced by logistic regression. Data processing and statistical analysis were performed using Statistical Package for the Social Sciences (version 22.0; SPSS, Chicago, IL, USA). A value of $\mathrm{P}<0.05$ was considered statistically significant.

\section{Results}

\section{General characteristics across BMD T-scores}

The general characteristics of the postmenopausal women with T2DM $(\mathrm{n}=214)$ are summarized in Table 1 . The mean age was $60.0(56.0-65.0)$ years, the average duration of T2DM was $10.0(4.0-15.0)$ years and the mean HbA1c (\%) level was 7.8 (6.8-9.3); the average BMDs at L2-4, FN and TH were $1.03 \pm 0.18,0.83 \pm 0.13$ and $0.89 \pm 0.14 \mathrm{~g} / \mathrm{cm}^{2}$, respectively. According to the BMD T-scores, subjects were divided into two groups: T-score $\geq-1$ (normal group) and T-score $<-1$ (osteoporosis/osteopenia group). As compared to subjects in T-score $\geq-1$ group, subjects in T-score $<-1$ group had significantly increased 10 -year probability of MOF and HF. The BMI, prevalence of biguanides treatment, serum total cholesterol and FT were significantly lower, while SHBG was markedly higher in T-score $<-1$ group than in T-score $\geq-1$ group.

\section{Correlations among sex hormones and BMDs, 10-year probability of MOF and $\mathrm{HF}$}

The associations of sex hormones with BMDs and 10-year probability of MOF and HF were determined by Spearman correlation analysis. Serum SHBG was inversely correlated with the BMDs at L2-4 $(\mathrm{r}=-0.287, \mathrm{P}<0.001)$, FN $(\mathrm{r}=-0.207, \mathrm{P}<0.001)$ and $\mathrm{TH}(\mathrm{r}=-0.284, \mathrm{P}<0.001)$ (Table 2 and Figure $1 A, B, C)$. While FT had a positive relationship with L2-4 BMD ( $\mathrm{r}=0.156, \mathrm{P}<0.05)$ (Table 2). Serum SHBG was also positively related to modified FRAX MOF $(\mathrm{r}=0.165, \mathrm{P}<0.05)$ and $\mathrm{HF}(\mathrm{r}=0.236, \mathrm{P}<0.001)$ (Table 2 and Figure $1 D, E)$.

The contributions of sex hormones to BMDs, MOF 
Table 1 General characteristics of postmenopausal women with T2DM according to BMD T-score

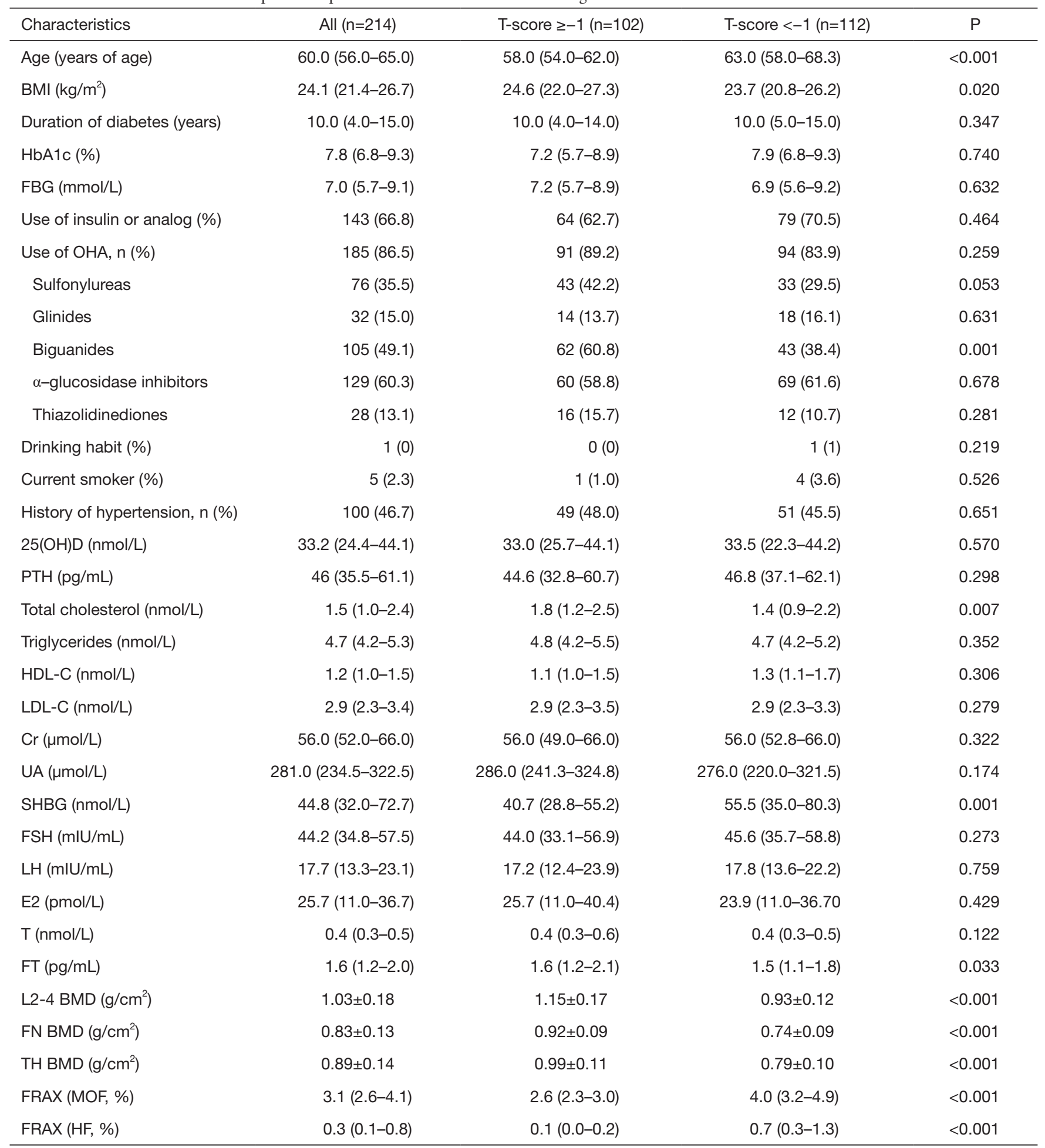

Data are presented as median (1st-4th quartiles) or mean \pm SD. BMI, body mass index; HbA1c, glycated hemoglobin A1C; FBG, fasting blood-glucose; OHA, oral hypoglycemic agents; PTH, parathyroid hormone; HDL-C, high-density lipoprotein cholesterol; LDL-C, lowdensity lipoprotein cholesterol; Cr, creatinine; UA, uric acid; SHBG, sex hormone-binding globulin; FSH, follicle-stimulating hormone; LH, luteinizing hormone; E2, estradiol; T, testosterone; FT, free testosterone; BMD, bone mineral density; L2-4, lumbar spine 2-4; FN, femoral neck; TH, total hip; FRAX, fracture risk algorithm; MOF, 10-year probability of major fracture; HF, 10-year probability of hip fracture. 
Table 2 Correlations of sex hormones with BMDs and modified FRAX in postmenopausal women with T2DM

\begin{tabular}{|c|c|c|c|c|c|c|}
\hline & SHBG & FSH & $\mathrm{LH}$ & E2 & $\mathrm{T}$ & FT \\
\hline L2-4 BMD & $-0.287^{\star *}$ & -0.017 & 0.028 & 0.075 & 0.112 & $0.156^{*}$ \\
\hline FN BMD & $-0.207^{\star *}$ & -0.001 & -0.025 & 0.006 & 0.074 & 0.073 \\
\hline TH BMD & $-0.284^{\star *}$ & -0.015 & -0.031 & 0.038 & 0.096 & 0.129 \\
\hline FRAX HF & $0.236^{\star *}$ & 0.017 & -0.008 & -0.029 & -0.037 & -0.089 \\
\hline
\end{tabular}

SHBG, sex hormone-binding globulin; FSH, follicle-stimulating hormone; LH, luteinizing hormone; E2, estradiol; T, testosterone; FT, free testosterone; BMD, bone mineral density; L2-4, lumbar spine; FN, femoral neck; TH, total hip; FRAX, fracture risk algorithm; MOF, 10-year probability of major fracture; HF, 10-year probability of hip fracture. ${ }^{* *}, \mathrm{P}<0.001 ;{ }^{*}, \mathrm{P}<0.05$.

A

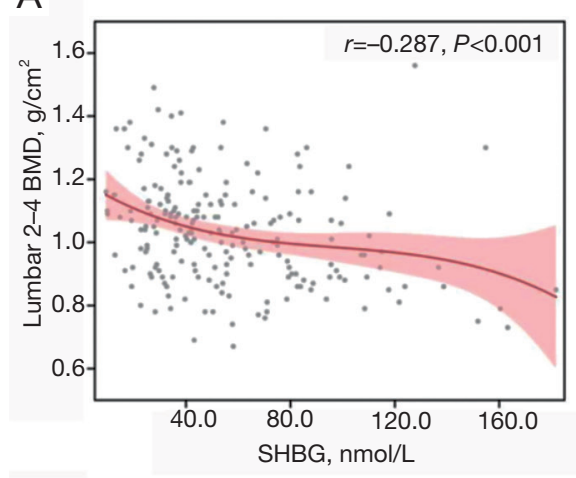

D

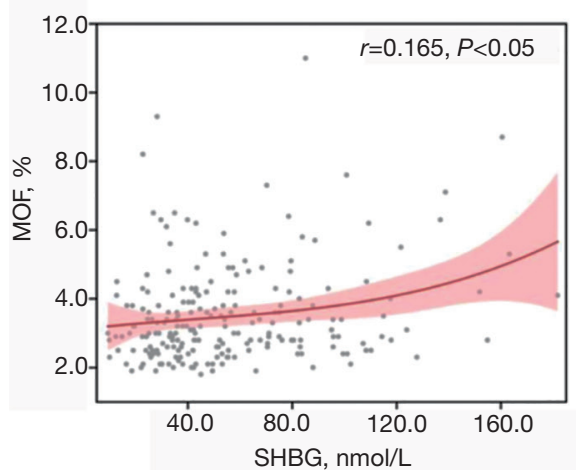

B

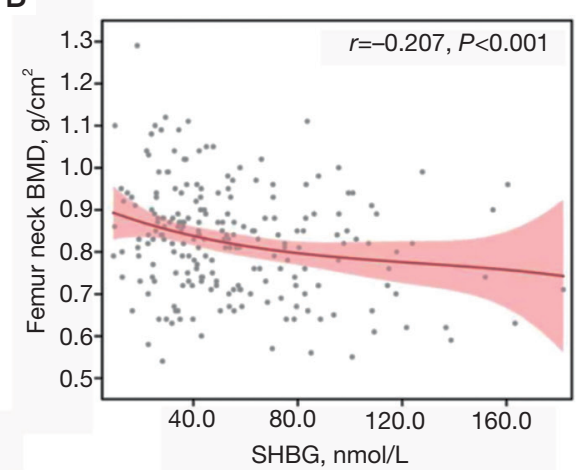

E

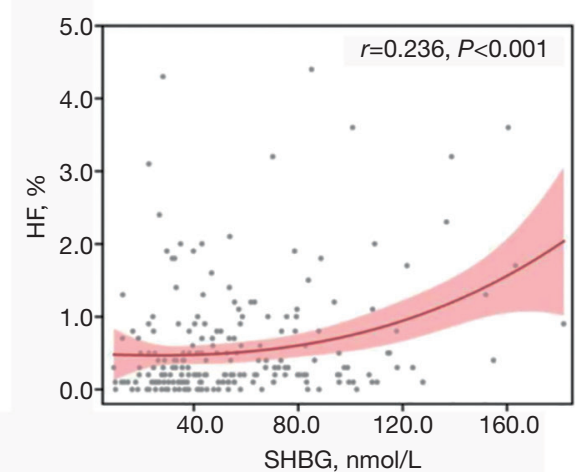

C

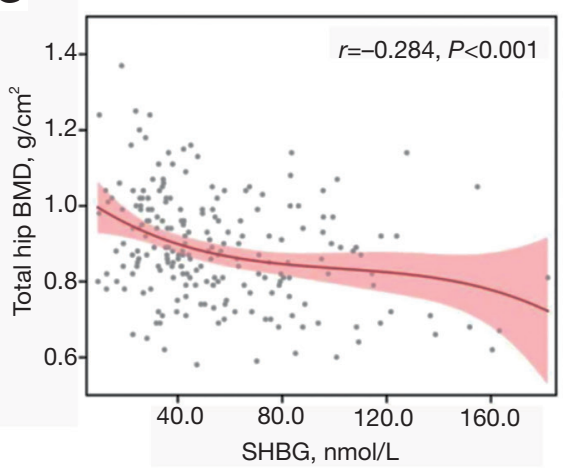

Figure 1 Correlations of serum SHBG with (A) BMD at lumbar 2-4; (B) BMD at femur neck; (C) BMD at total hip; (D) modified FRAX MOF; (E) modified FRAX HF in postmenopausal women with T2DM. SHBG, sex hormone-binding globulin; BMD, bone mineral density; FRAX, fracture risk algorithm; MOF, 10-year probability of major fracture; HF, 10-year probability of hip fracture.

and HF in postmenopausal women with T2DM were further analyzed by multivariate linear regression analysis. BMDs at three sites, MOF and HF were used as dependent variables, and sex hormones as independent variables. In unadjusted model (Model 1), SHBG and E2 were the determinants of BMDs at three sites (Table 3). However, after adjustment for age, duration of diabetes, HbA1c, FBG, insulin or analog use, OHA use (sulfonylureas, glinides, biguanides, $\alpha$-glucosidase inhibitors, and thiazolidinediones respectively), history of hypertension, $\mathrm{PTH}, 25(\mathrm{OH}) \mathrm{D}$, 
Table 3 Sex hormones related to BMDs and modified FRAX in postmenopausal women with T2DM

\begin{tabular}{|c|c|c|c|c|c|c|c|c|c|c|c|}
\hline \multirow{2}{*}{ Items } & \multicolumn{3}{|c|}{ L2-4 BMD } & \multicolumn{3}{|c|}{ FN BMD } & \multicolumn{3}{|c|}{ TH BMD } & \multicolumn{2}{|c|}{ FRAX } \\
\hline & $\beta(\mathrm{P})$ & $\beta(P)$ & $\beta(P)$ & $\beta(\mathrm{P})$ & $\beta(P)$ & $\beta(P)$ & $\beta(P)$ & $\beta(P)$ & $\beta(P)$ & $\beta(P)$ & $\beta(P)$ \\
\hline SHBG & $-0.244^{\star \star}$ & $-0.228^{*}$ & $-0.199^{*}$ & $-0.253^{\star *}$ & $-0.209^{*}$ & -0.120 & $-0.311^{\star \star}$ & $-0.313^{\star \star}$ & $-0.233^{*}$ & $0.253^{\star \star}$ & $0.262^{\star \star}$ \\
\hline FSH & 0.028 & -0.131 & -0.096 & 0.046 & -0.040 & 0.024 & 0.082 & -0.003 & 0.063 & -0.136 & -0.091 \\
\hline E2 & $0.154^{*}$ & 0.083 & 0.079 & $0.145^{\star}$ & 0.057 & 0.054 & $0.147^{\star}$ & 0.083 & 0.079 & $-0.159^{\star}$ & -0.097 \\
\hline $\mathrm{T}$ & 0.014 & 0.091 & 0.105 & 0.036 & 0.098 & 0.149 & 0.036 & 0.137 & $0.179^{\star}$ & -0.016 & 0.021 \\
\hline FT & 0.008 & -0.043 & -0.048 & -0.070 & -0.093 & -0.133 & -0.016 & -0.099 & -0.128 & 0.033 & -0.029 \\
\hline
\end{tabular}

L2-4, lumbar spine; FN, femoral neck; TH, total hip; BMD, bone mineral density; FRAX, Fracture Risk Algorithm; MOF, 10-year probability of major fracture; HF, 10-year probability of hip fracture; SHBG, sex hormone-binding globulin; FSH, follicle-stimulating hormone; LH, luteinizing hormone; E2, estradiol; T, testosterone; FT, free testosterone; BMI, body mass index; HbA1c, glycated hemoglobin A1C; FBG, fasting blood-glucose; OHA, oral hypoglycemic agents; PTH, parathyroid hormone; HDL-C, high-density lipoprotein cholesterol; LDL-C, low-density lipoprotein cholesterol; Cr, creatinine; UA, uric acid. Model 1: unadjusted model. Model 2: adjustment for age, duration of diabetes, HbA1c, FBG, insulin or analog use, OHA use (sulfonylureas, glinides, biguanides, $\alpha$-glucosidase inhibitors, and thiazolidinediones respectively), history of hypertension, $\mathrm{PTH}, 25(\mathrm{OH}) \mathrm{D}$, total cholesterol, triglyceride, HDL-C, LDL-C, Cr and UA. Model 3: adjustment for age, duration of diabetes, HbA1c, FBG, use of insulin or analog, use of OHA (sulfonylureas, glinides, biguanides, $\alpha$-glucosidase inhibitors, and thiazolidinediones respectively), history of hypertension, $\mathrm{PTH}, 25(\mathrm{OH}) \mathrm{D}$, total cholesterol, triglyceride, HDL-C, LDL-C, Cr, UA and BMI. **, $\mathrm{P}<0.001 ;{ }^{*}, \mathrm{P}<0.05$.

total cholesterol, triglyceride, HDL-C, LDL-C, Cr and UA in Model 2, SHBG was still a determinant of BMDs at L2 -4 $(\beta=-0.228, \mathrm{P}<0.05), \mathrm{FN}(\beta=-0.209, \mathrm{P}<0.05)$ and $\mathrm{TH}$ $(\beta=-0.313, \mathrm{P}<0.05)$, but $\mathrm{E} 2 \mathrm{had}$ no relationship with the BMDs (Table 3). After further adjustment for BMI, SHBG was still related to the BMD at L2-4 and TH although its contribution reduced, but there was no relationship between SHBG and BMD at FN. SHBG and E2 were the determinants of modified MOF (SHBG, $\beta=0.253$, $\mathrm{P}<0.001$; $\mathrm{E} 2, \beta=-0.159, \mathrm{P}<0.05)$; SHBG was the only determinant of modified HF $(\beta=0.262, \mathrm{P}<0.001)$. Besides, we also assessed the associations among sex hormones and 10-year probability of fractures evaluated by standard FRAX tool (without replacing RA with DM), the results were similar to the present study showed (Table S1).

\section{Determinants of osteoporosis/osteopenia}

To confirm the contribution of SHBG to BMDs, logistic regression was employed to examine the ORs of osteoporosis/osteopenia according to the per SD increase in important sex hormones including SHBG, E2, T and FT. As shown in Table 4, after adjustment for multi-variables including age, BMI, duration of diabetes, HbA1c, FBG, use of insulin or analog, use of OHA (sulfonylureas, glinides, biguanides, $\alpha$-glucosidase inhibitors, and thiazolidinediones respectively), history of hypertension, $\mathrm{PTH}, 25(\mathrm{OH}) \mathrm{D}$, total cholesterol, triglyceride, HDL-C, LDL-C, Cr and $\mathrm{UA}$, the per SD increase in SHBG caused a $2 \%$ increase in the risk for osteoporosis/osteopenia $(\mathrm{OR}=1.02,95 \% \mathrm{CI}$ : $1.00-1.04, \mathrm{P}=0.017)$. After further adjustment for $\mathrm{E} 2$ or $\mathrm{T}$, the per SD increase in SHBG still caused a $2 \%$ increase in the risk for osteoporosis/osteopenia. Moreover, E2 and T showed no association with osteoporosis/osteopenia.

The prevalence of osteoporosis/osteopenia showed an increased trend with the increase in SHBG of postmenopausal women with T2DM (P trend=0.001) (Figure 2 and Table 5). Logistic regression analysis revealed that, compared with subjects in Q-1, subjects in Q-4 had increased risk for osteoporosis/osteopenia ( $\mathrm{OR}=4.21,95 \%$ CI: $1.17-15.12, \mathrm{P}=0.015)$ even after adjustment for multivariables including $\mathrm{E} 2$ and $\mathrm{T}$ (Table 5).

To further explore the determinants of osteoporosis/ osteopenia, multivariate logistic regression model with independent variables including SHBG and potential risk factors was employed. The ROC of osteoporosis/ osteopenia was produced to identify the cut-off values of SHBG associated with the maximal Youden index (Figure S1). According to the cutoffs, SHBG was divided into higher $(>53.4 \mathrm{nmol} / \mathrm{L})$ and normal $(\leq 53.4 \mathrm{nmol} / \mathrm{L})$ 
Table 4 ORs of osteoporosis/osteopenia for per SD increase in SHBG, FSH or E2 of postmenopausal women with T2DM

\begin{tabular}{|c|c|c|c|c|c|c|}
\hline Times & \multicolumn{2}{|c|}{ SHBG } & \multicolumn{2}{|l|}{ E2 } & \multicolumn{2}{|l|}{$\mathrm{T}$} \\
\hline Unadjusted & $1.02(1.01-1.03)$ & $<0.001$ & $1.00(0.99-1.00)$ & 0.087 & $0.52(0.15-1.77)$ & 0.295 \\
\hline MV adjusted ${ }^{a}$ & $1.02(1.00-1.04)$ & 0.017 & $0.99(0.98-1.01)$ & 0.231 & $0.22(0.04-1.30)$ & 0.095 \\
\hline MV adjusted ${ }^{\mathrm{a}}$ plus SHBG & - & - & $0.99(0.98-1.01)$ & 0.238 & $0.21(0.04-1.30)$ & 0.094 \\
\hline MV adjusted ${ }^{\mathrm{a}}$ plus $\mathrm{T}$ & $1.02(1.00-1.04)$ & 0.018 & $0.99(0.98-1.00)$ & 0.196 & - & - \\
\hline
\end{tabular}

SD, standard deviation; OR, odds ratio; Cl, confidence interval; SHBG, sex hormone-binding globulin; E2; estradiol; T, testosterone; BMI, body mass index; HbA1c, glycated hemoglobin A1C; FBG, fasting blood-glucose; OHA, oral hypoglycemic agents; PTH, parathyroid hormone; HDL-C, high-density lipoprotein cholesterol; LDL-C, low-density lipoprotein cholesterol; Cr, creatinine; UA, uric acid. a, Multivariable regression model with adjustment for age, BMI, duration of diabetes, HbA1c, FBG, use of insulin or analog, use of OHA (sulfonylureas, glinides, biguanides, $\alpha$-glucosidase inhibitors, and thiazolidinediones respectively), history of hypertension, $\mathrm{PTH}, 25(\mathrm{OH}) \mathrm{D}$, total cholesterol, triglyceride, HDL-C, LDL-C, Cr and UA.

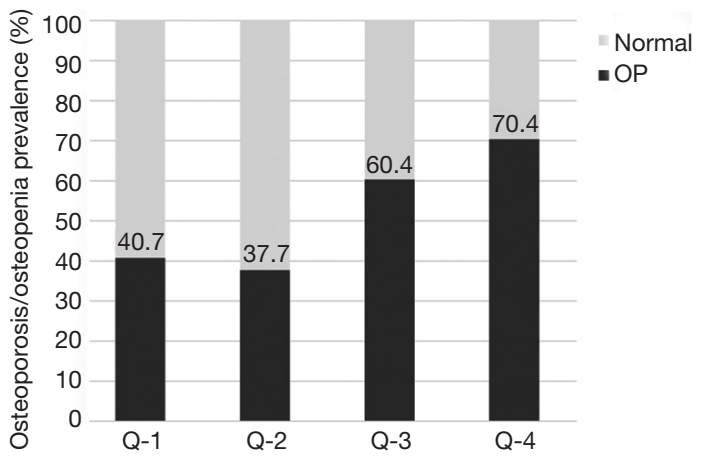

Figure 2 Prevalence rate of osteoporosis/osteopenia according to SHBG quartiles in postmenopausal women with T2DM. Q-1: $<32.3 \mathrm{nmol} / \mathrm{L}, \mathrm{Q}-2: 32.3-44.8 \mathrm{nmol} / \mathrm{L}, \mathrm{Q}-3: 44.9-73.0 \mathrm{nmol} /$ L, Q-4: >73.0 nmol/L. SHBG, sex hormone-binding globulin; T2DM, type 2 diabetes mellitus. Normal, normal bone mineral density (T-score $\geq-1$ ), OP, osteoporosis/osteopenia (T-score <-1).

levels. Forest plot showed that higher SHBG $(\mathrm{OR}=2.89$, 95\% CI: $1.26-6.63, \mathrm{P}=0.012)$ and per year increase of age $(\mathrm{OR}=1.12,95 \% \mathrm{CI}: 1.04-1.20, \mathrm{P}=0.002)$ were the risk factors of osteoporosis/osteopenia, while per unit increase in $\mathrm{BMI}(\mathrm{OR}=0.87,95 \% \mathrm{CI}: 0.76-0.99, \mathrm{P}=0.032)$ was a protective factor (Figure 3).

\section{Discussion}

In the present study, the primary findings were that, in postmenopausal women with T2DM, SHBG was inversely associated with the BMDs at L2-4, FN and TH, but positively with the 10 -year probability of MOF and HF; high SHBG was the major risk factor of osteoporosis/ osteopenia independent of E2 and T; FSH, LH, E2, T or FT was not associated with the BMDs, MOF or HF. To our knowledge, our study for the first time investigated the relationships of serum SHBG with BMDs and future risk of fractures in postmenopausal women with T2DM.

Available studies exploring the associations among sex hormones, BMDs and fracture risk in postmenopausal women mainly focus on non-diabetes subjects. Some studies indicate that high serum SHBG and low T (not E2) correlate with increased risk of hip fractures in the general postmenopausal women, some report that serum E2 and SHBG (not T) are related to the BMDs in women aged over 65 years, and others reveal E2 and T (not SHBG) are the determinant of BMD in postmenopausal women $(5,17,18)$. Our study investigated the relationships of hormones with BMD and fracture risk in postmenopausal women with T2DM, and results revealed that the serum SHBG was related to the BMDs at three sites and fracture risk determined with FRAX, while neither $\mathrm{E} 2$ nor $\mathrm{T}$ had relationships with the BMDs or fracture risk assessed with FRAX.

It has universally been acknowledged that estrogen deficiency plays a central role in the pathogenesis of postmenopausal osteoporosis (19). In our diabeticpostmenopausal individuals, E2 was found as a determinant of BMDs in unadjusted regression model (Table 3), but its significance disappeared after adjustment for multi-variables. This might be explained as that the postmenopausal individuals in the present study had an extensive E2 deficiency, and thus the protective effect of E2 
Table 5 ORs of osteoporosis/osteopenia for quartiles of SHBG in postmenopausal women with T2DM

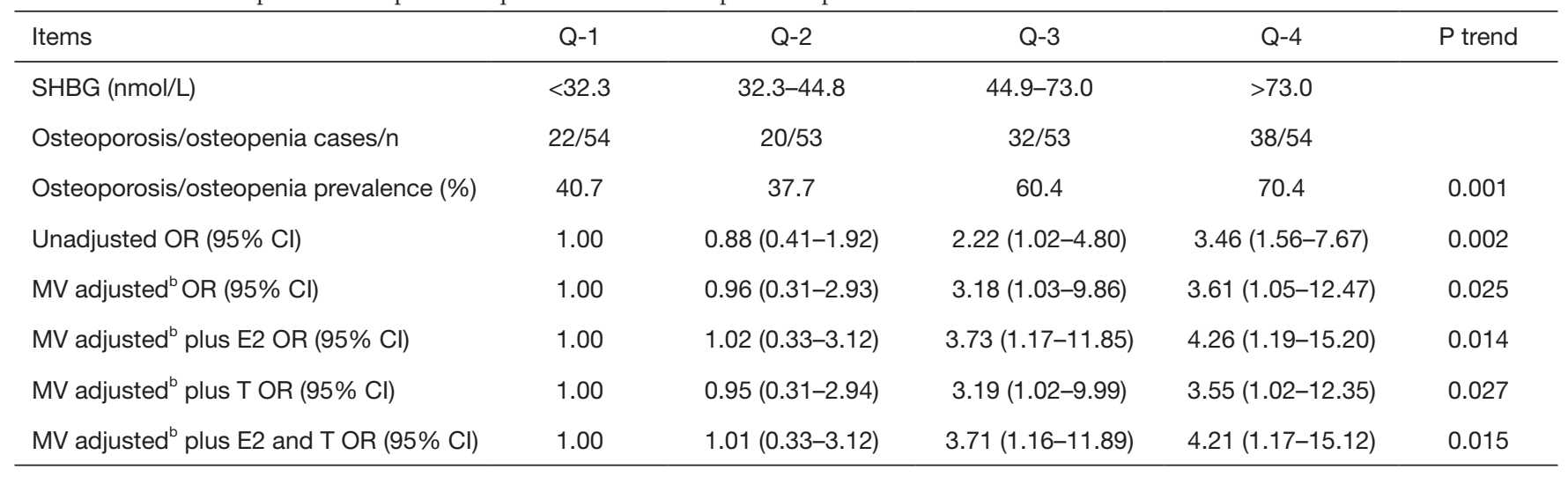

SHBG, sex hormone-binding globulin; OR, odds ratio; Cl, confidence interval; BMI, body mass index; HbA1c, glycated hemoglobin A1C; FBG, fasting blood-glucose; OHA, oral hypoglycemic agents; PTH, parathyroid hormone; HDL-C, high-density lipoprotein cholesterol; LDL-C, low-density lipoprotein cholesterol; Cr, creatinine; UA, uric acid. ${ }^{b}$, Multivariable regression analysis with adjustment for age, BMI, duration of diabetes, HbA1c, FBG, use of insulin or analog, use of OHA (sulfonylureas, glinides, biguanides, $\alpha$-glucosidase inhibitors, and thiazolidinediones respectively), history of hypertension, PTH, 25(OH)D, total cholesterol, triglyceride, HDL-C, LDL-C, Cr and UA.

on BMDs was quite weak. The associations identified in the unadjusted model might be attributed to the interactions with other potential factors such as age and BMI.

SHBG, a transport protein, acts to bind main sex hormones including circulating $\mathrm{T}$ and $\mathrm{E} 2$, transporting them towards target cells. Its effect on sex steroids bioactivity and metabolic outcomes are widely investigated $(20,21)$. A widely accepted theory about how SHBG is involved in bone metabolism is based on its anti-estrogenic effect. Higher SHBG binds to estrogen to reduce its biologically active form, which consequently reduces $\mathrm{BMD}$ and increases fracture risk (22). However, there is evidence showing that SHBG is not only a carrier protein that affects the bone indirectly, but also a mediator that specifically modifies the membrane receptors including estrogen receptors and consequently triggers the relevant intracellular signaling pathways $(23,24)$. The present study revealed that high SHBG was an independent risk factor of osteoporosis/osteopenia even after adjustment for E2 and T. Even though the biological mechanism of how SHBG is involved into diabetic bone and fractures remained unclear, our findings still suggest that measurement of SHBG could give a clue to the risk of osteoporosis/osteopenia and future fracture risk in postmenopausal women with T2DM.

Notably, in our results, the associations of SHBG with BMDs at L2-4 and TH were compromised and its association with $\mathrm{FN}$ disappeared after adjustment for BMI. One possible explanation is that BMI is one of numerous factors that affect BMD (25). Indeed, our study also demonstrated that BMI was a protective factor of osteoporosis/osteopenia as shown by multivariate logistic regression analysis (Figure 3). In addition, studies also reveal that BMI may markedly influence the variation of SHBG. Higher BMI has been found to be associated with lower SHBG, leading to the elevation of active E2 and T, which results in the maintenance or increase of BMD (21). Despite these findings, our study further revealed that SHBG was still an independent risk factor of osteoporosis/osteopenia in multivariable regression model, even after adjustment for BMI.

This study has several limitations: (I) this study was retrospective, and the changes in sex hormones and BMDs over time were not examined. Thus, whether their changes are involved in the interactions is still unclear; (II) although several osteoporotic fractures were recorded as longterm end points, the number of fractures was too small to conduct statistical analysis. Therefore, the modified FRAX was used to calculate the 10-year probability of HF and MOF. The replacement of RA with diabetes in FRAX improves the performance of FRAX in assessing fracture risk of diabetes patients; (III) no healthy controls were included in the present study.

In conclusion, our study suggests that, in postmenopausal women with T2DM, higher serum SHBG was associated with lower BMDs, higher risk for osteoporosis/ osteopenia and greater future fracture risk determined by FRAX. Longitudinal clinical studies are needed to confirm the role of SHBG in the bone health of postmenopausal 


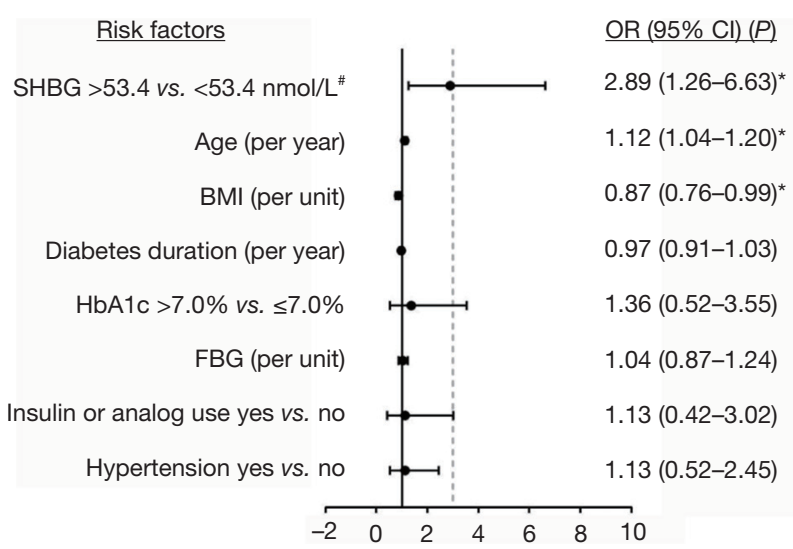

Figure 3 Forest plot of ORs for potential determinants of osteoporosis/osteopenia in postmenopausal women with T2DM. Logistic regression model with adjustment for use of $\mathrm{OHA}$ (sulfonylureas, glinides, biguanides, $\alpha$-glucosidase inhibitors, and thiazolidinediones respectively), PTH, 25(OH)D, total cholesterol, triglyceride, HDL-C, LDL-C, Cr and UA. *, the cut-off values were determined by the maximal Youden index of receiver operating characteristic curves (ROC). * $\mathrm{P}<0.05$. OR, odds ratio; CI, confidence interval; T2DM, type 2 diabetes mellitus; SHBG, sex hormone-binding globulin; BMI, body mass index; HbAlc, glycated hemoglobin A1C; FBG, fasting blood-glucose; OHA, oral hypoglycemic agents; PTH, parathyroid hormone; HDL-C, highdensity lipoprotein cholesterol; LDL-C, low-density lipoprotein cholesterol; Cr, creatinine; UA, uric acid.

women with T2DM.

\section{Acknowledgments}

Funding: This study was supported by the Shanghai Health and Family Planning Commission (No. 201540166) and the Chinese National Natural Science Foundation (No. 81370018 and No.81570796).

\section{Footnote}

Conflicts of Interest: The authors have no conflicts of interest to declare.

Ethical Statement: The authors are accountable for all aspects of the work (if applied, including full data access, integrity of the data and the accuracy of the data analysis) in ensuring that questions related to the accuracy or integrity of any part of the work are appropriately investigated and resolved. The protocol for the research project was approved by the
Ethics Committee of Rui-jin Hospital affiliated to Shanghai Jiao-tong University School of Medicine (2019 No. 45). All procedures performed in studies were in accordance with the ethical standards of the Institutional Research Board and with the Helsinki Declaration.

\section{References}

1. Watts NB, investigators G. Insights from the Global Longitudinal Study of Osteoporosis in Women (GLOW). Nat Rev Endocrinol 2014;10:412-22.

2. Compston JE, McClung MR, Leslie WD. Osteoporosis. Lancet 2019;393:364-76.

3. Black DM, Rosen CJ. Clinical Practice. Postmenopausal Osteoporosis. N Engl J Med 2016;374:254-62.

4. Bjornerem A, Ahmed LA, Joakimsen RM, et al. A prospective study of sex steroids, sex hormone-binding globulin, and non-vertebral fractures in women and men: the Tromso Study. Eur J Endocrinol 2007;157:119-25.

5. Lee JS, LaCroix AZ, Wu L, et al. Associations of serum sex hormone-binding globulin and sex hormone concentrations with hip fracture risk in postmenopausal women. J Clin Endocrinol Metab 2008;93:1796-803.

6. Shanbhogue VV, Mitchell DM, Rosen CJ, et al. Type 2 diabetes and the skeleton: new insights into sweet bones. Lancet Diabetes Endocrinol 2016;4:159-73.

7. Majima T, Komatsu Y, Yamada T, et al. Decreased bone mineral density at the distal radius, but not at the lumbar spine or the femoral neck, in Japanese type 2 diabetic patients. Osteoporos Int 2005;16:907-13.

8. Melton LJ, 3rd, Leibson CL, Achenbach SJ, et al. Fracture risk in type 2 diabetes: update of a population-based study. J Bone Miner Res 2008;23:1334-42.

9. Zhou Y, Li Y, Zhang D, et al. Prevalence and predictors of osteopenia and osteoporosis in postmenopausal Chinese women with type 2 diabetes. Diabetes Res Clin Pract 2010;90:261-9.

10. Chen FP, Kuo SF, Lin YC, et al. Status of bone strength and factors associated with vertebral fracture in postmenopausal women with type 2 diabetes. Menopause 2019;26:182-8.

11. Kanis JA, Oden A, Johnell O, et al. The use of clinical risk factors enhances the performance of BMD in the prediction of hip and osteoporotic fractures in men and women. Osteoporos Int 2007;18:1033-46.

12. Semel J, Gray JM, Ahn HJ, et al. Predictors of outcome following hip fracture rehabilitation. PM R 2010;2:799-805. 
13. Reistetter TA, Graham JE, Deutsch A, et al. Diabetes comorbidity and age influence rehabilitation outcomes after hip fracture. Diabetes Care 2011;34:1375-7.

14. Carnevale V, Morano S, Fontana A, et al. Assessment of fracture risk by the FRAX algorithm in men and women with and without type 2 diabetes mellitus: a cross-sectional study. Diabetes Metab Res Rev 2014;30:313-22.

15. Schwartz AV, Vittinghoff E, Bauer DC, et al. Association of BMD and FRAX score with risk of fracture in older adults with type 2 diabetes. JAMA 2011;305:2184-92.

16. Greendale GA, Lee NP, Arriola ER. The menopause. Lancet 1999;353:571-80.

17. Rapuri PB, Gallagher JC, Haynatzki G. Endogenous levels of serum estradiol and sex hormone binding globulin determine bone mineral density, bone remodeling, the rate of bone loss, and response to treatment with estrogen in elderly women. J Clin Endocrinol Metab 2004;89:4954-62.

18. Lambrinoudaki I, Christodoulakos G, Aravantinos L, et al. Endogenous sex steroids and bone mineral density in healthy Greek postmenopausal women. J Bone Miner Metab 2006;24:65-71.

Cite this article as: Jing Y, Wang X, Yu J, Wang X, Zhou Y, Tao B, Sun L, Liu J, Zhao H. Associations of serum sex hormone binding globulin with bone mineral densities and higher 10-year probability of fractures in postmenopausal women with type 2 diabetes mellitus. Ann Transl Med 2019;7(18):457. doi: 10.21037/atm.2019.08.46
19. Compston JE. Sex steroids and bone. Physiol Rev 2001;81:419-47.

20. Laurent MR, Helsen C, Antonio L, et al. Effects of sex hormone-binding globulin (SHBG) on androgen bioactivity in vitro. Mol Cell Endocrinol 2016;437:280-91.

21. Liu S, Sun Q. Sex differences, endogenous sex-hormone hormones, sex-hormone binding globulin, and exogenous disruptors in diabetes and related metabolic outcomes. J Diabetes 2018;10:428-41.

22. Legrand E, Hedde C, Gallois Y, et al. Osteoporosis in men: a potential role for the sex hormone binding globulin. Bone 2001;29:90-5.

23. Fortunati N. Sex hormone-binding globulin: not only a transport protein. What news is around the corner? J Endocrinol Invest 1999;22:223-34.

24. Kahn SM, Hryb DJ, Nakhla AM, et al. Sex hormonebinding globulin is synthesized in target cells. J Endocrinol 2002;175:113-20.

25. De Laet C, Kanis JA, Oden A, et al. Body mass index as a predictor of fracture risk: a meta-analysis. Osteoporos Int 2005;16:1330-8. 


\section{Supplementary}

Table S1 Sex hormones related to standard FRAX in postmenopausal women with T2DM

\begin{tabular}{|c|c|c|c|c|c|c|c|c|}
\hline Items & \multicolumn{4}{|c|}{ Standard MOF } & \multicolumn{4}{|c|}{ Standard HF } \\
\hline SHBG & 0.008 & 0.002 & 0.247 & 0.001 & 0.005 & 0.001 & 0.265 & $<0.001$ \\
\hline FSH & -0.009 & 0.006 & -0.153 & 0.130 & -0.003 & 0.003 & -0.096 & 0.343 \\
\hline LH & -0.008 & 0.011 & -0.065 & 0.492 & -0.005 & 0.006 & -0.073 & 0.443 \\
\hline $\mathrm{T}$ & -0.060 & 0.375 & -0.012 & 0.872 & 0.062 & 0.217 & 0.022 & 0.774 \\
\hline FT & 0.058 & 0.143 & 0.032 & 0.685 & -0.025 & 0.082 & -0.024 & 0.766 \\
\hline
\end{tabular}

FRAX, fracture risk algorithm; MOF, major fracture; HF, hip fracture; SHBG, sex hormone binding globulin; FSH, follicle-stimulating hormone; LH, luteinizing hormone; E2, estradiol; T, testosterone; FT, free testosterone.

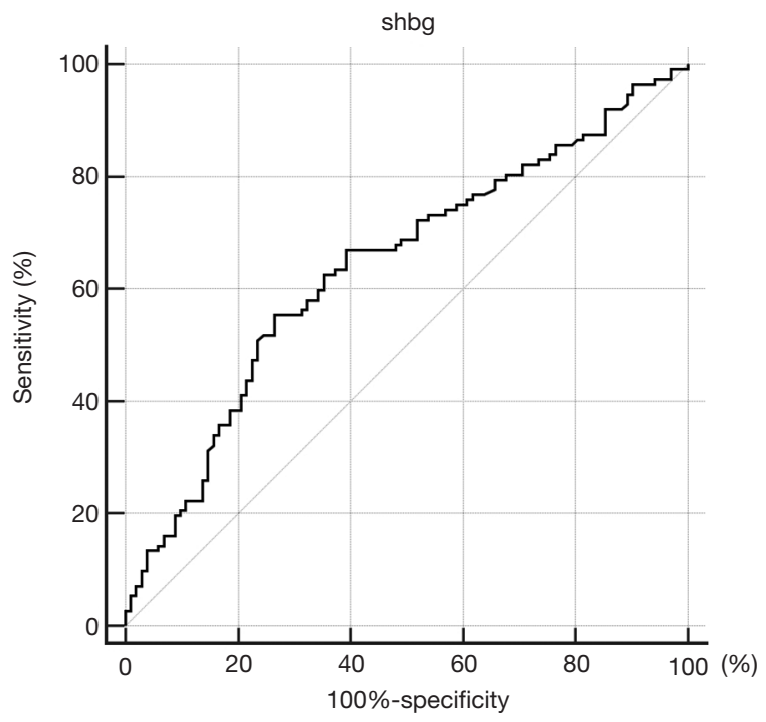

Figure S1 Receiver operating characteristic curves (ROC) of osteoporosis/osteopenia. Area under the ROC curve (AUC) $=0.640$. Significance level $\mathrm{P}=0.0002$. Youden Index associated criterion: $>53.4 \mathrm{nmol} / \mathrm{L}$. Sensitivity $=55.36 \%$, Specificity $=73.53 \%$. SHBG, sex hormone-binding globulin. 